\title{
Evaluating the Users' Satisfaction Using Inclusive Initiatives in Two Different Environments: The University and a Research Conference
}

\author{
Ana Iglesias ${ }^{1}$, Lourdes Moreno ${ }^{1}$, Javier Jiménez ${ }^{2}$, and Pablo Revuelta ${ }^{2}$ \\ ${ }^{1}$ Computer Science Department, Universidad Carlos III de Madrid, \\ Avda. Universidad, 30 - 28911 Leganés (Madrid), Spain \\ \{aiglesia, lmoreno\}@inf.uc3m.es \\ ${ }^{2}$ Spanish Centre of Captioning and Audio Description, \\ Avda. Gregorio P. Barba, 1 - 28918 Leganés (Madrid), Spain \\ \{jjdorado, prevuelta\} @cesya.es
}

\begin{abstract}
This paper presents evaluation results of the user's satisfaction using the APEINTA project which main aim is to provide accessibility in education, in and out of the classroom. APEINTA is the Spanish acronym for "Aiming for an Inclusive Education based on Assistive Technology". The APEINTA project is focused in two main inclusive proposals: first, it deals with eliminating hard of hearing students' communication barriers in the classroom, providing them automatic real-time captioning and other mechanisms for making easy the communication with the teacher and others students; and second, it deals also in providing an accessible Web learning platform with accessible digital resources, so every student can access them in and out of the classroom.
\end{abstract}

Keywords: Inclusion, Web Accessibility, E-learning, Special Needs, Assistive Technology, Real-time Captioning.

\section{Introduction}

Historically, students with disabilities have experienced inadequate access to lecture materials in the classroom, and insufficient access to the academic resources necessary to sustain their progress. Sign Language interpreters have been traditionally used during the class for deaf students' inclusion. However, nowadays not every student with hearing disability uses Sign Language, thus signing does not provide deaf students full access to classroom information [1]. Nowadays, some researchers are working in lectures transcription based on Automatic Speech Recognition (ASR).

The Liberated Learning Project (LLP) is one of the most active initiatives, collaboration with IBM in developing the ViaScribe software based on the ASR ViaVoice (VV) software [2].

On the other hand, several research groups and organizations developed technologies and standards related to how to achieve accessible educational resources and how to develop Web platforms. For instance, the guidelines for developing accessible learning applications (IMS) [3], the World Wide Web Consortium (WC3) [4] and the 
Web Accessibility Initiative WAI [5] which play a leading role in promoting the importance of accessibility and developing guidelines which can help when developing accessible Web resources. These guidelines are useful for developing accessible Web resources.

There are other technologies as Learning Management Systems (LMS) or Learning Content Management Systems (LCMS) that include accessibilities issues as the dotLRN LMS [6] or the Atutor LCMS [7].

In this paper we evaluate the user's satisfaction using the APEINTA inclusive tools in two different environments: the University and a Research Conference.

\section{The APEINTA Architecture}

The APEINTA architecture shown in Figure 1 presents two well differentiated applications: a real-time captioning (using ASR technologies) and synthetic speaking multisystem (using Text-to-Speech technologies, TTS) in the classroom [8] in order to avoid communication barriers for hearing impaired students and students with speaking problems (see section 3); and a Web platform with accessible digital contents that can be used in and out of the classroom for students of all abilities, including benefits in their learning [9].

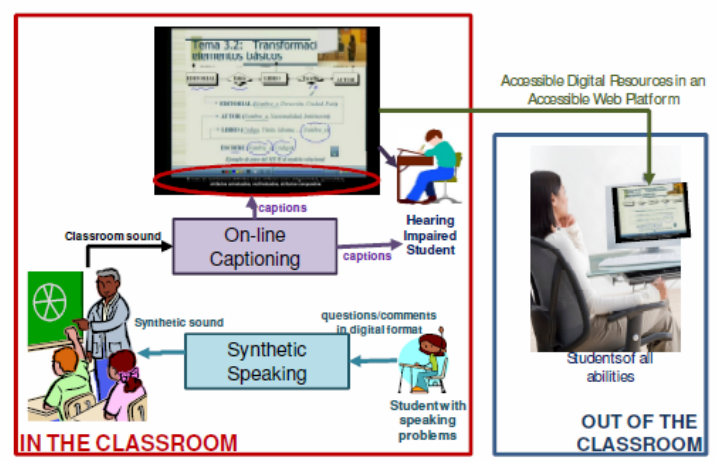

Fig. 1. APEINTA architecture

Firstly, the APEINTA project was designed and implemented in the educational environment (at University), but after the first evaluation process we realized the benefits that this initiative provide to people with temporal or permanent disabilities, older adults, foreign people or students with speaking problems, for instance. Subsequently this project was introduced and evaluated too during a talk in a research conference.

\section{Evaluation of APEINTA}

The evaluation of the users' satisfaction using APEINTA was carried out by surveying the users after using the inclusive tools. 
The ASR and TTS services of APEINTA have been evaluated in 2009 during different classes of 50 minutes. These classes belong to Database Design signature in 3rd course of Computer Science degree at the Carlos III University of Madrid. Moreover, the APEINTA services were also evaluated during a 30 minutes talk in the ACAPPS (Federation of Families and Deaf People of Catalonia) Congress.

For the ASR evaluation, the orator trained the ASR software (Dragon NaturallySpeaking v.10) during 40 minutes approximately. The training was conducted by reading predefined texts ( 30 minutes) and adding new words to the vocabulary according to the subject specific vocabulary (10 minutes).

During the evaluation session (in both environments), the users could receive the captioning in a PDA, a Laptop and in a big screen in the auditorium (which size was calculated according to the size of the auditorium in each environment) overlapped with the lecture slides connected to the server. Moreover, they could use the PDA and the Laptop in order to write questions and comments and use the TTS service.

Five deaf and hard of hearing volunteers and two hearing volunteers participated in the evaluation of APEINTA during the conference talk. Moreover, 45 hearing students evaluated the APEINTA initiative in the classroom, 10 of them simulating hearing disabilities. Finally, three experts in captioning, usability and accessibility also evaluated the system in the classroom.

For this evaluation, surveys were distributed to the participants. All of them completed the survey voluntarily and some of them provided written comments about improvements and satisfaction to the inclusive initiatives. On the survey, the questions were measured using a 1 to 5 Likert-type scale [10], with 1 representing Completely Disagree and 5 representing Completely Agree. Furthermore, the experts in captioning, usability and accessibility were interviewed to gain a richer understanding of the feedback.

All participants were really satisfied with the pedagogical initiative (all of them answering 5 in the surveys). Related to the captioning service, most of them were globally satisfied with the captioning service. The average was 4.9 and the standard deviation was 0.37 . Although the speech transcription was not completely correct (the average Word-Error-Rate measured was $10.4 \%$ during both sessions according to the Levenshtein Edit Distance) most of the participants answered that they could detect the errors and they could understand the global sense of the sentence (average of 4.55 and standard deviation of 0.79). Moreover, all of them were satisfied with the TTS service and the hearing students answered that the use of these technologies had not negative influence in their learning process.

On the other hand, the accessible Website including accessible pedagogical resources was evaluated in 2009 at University, but it was not implemented for the ACCAPS congress. One hundred eighty-nine students participated in the survey (90 of them interacting with the accessible website), two persons with audio and visual disabilities and an accessibility human expert. The survey of the system showed how the accessible website presents pedagogical benefits to all the students and allows students who cannot assist to the classroom to pass the final exam of the subject. Additionally, the survey and the system monitoring showed that the system and its multimedia resources were accessible to all the students. It is important to underline that the e-learning platform and every interface used by the students in the APEINTA 
project offers a universal access for any typology of users, so Inclusive Methodologies and Universal Design Principles have been taken into account.

\section{Conclusions and Further Research}

The APEINTA project proposes to use two different inclusive proposals. In the classroom, the use of ASR and TTS services allows to avoid communication barriers among the speaker and the listeners. Out of the classroom, the use of accessible Websites and accessible digital resources provide benefits to all the users.

This paper presents evaluation results of the user's satisfaction using the APEINTA initiatives in two different environments: at University and during a talk in a Conference. Nowadays, we are working in evaluating the APEINTA project in a course where students with different levels of disabilities are enrolled and introducing these inclusive initiatives in other environments.

\section{References}

1. Marschark, M., Sapere, P., Convertino, C., Seewagen, R.: Access to Postsecondary Education through Sign Language Interpreting. The Journal of Deaf Studies and Deaf Education 10, 38-50 (2005)

2. Bain, K., Basson, S., Wald, M.: Speech Recognition in University Classrooms: Liberated Learning Project. In: Proc. of 5th Annual International ACM Conference on Assistive Technologies, pp. 192-196 (2005)

3. IMS guidelines for developing accessible learning applications, version 1.0 (2002), http: / /www.imsglobal org/accessibility

4. World Wide Web Consortium, http: / /www .w3 . org/

5. Web Accessibility Initiative (WAI), http: / / www . w3 . org/WAI/

6. dotLRN LMS (Learning Management System), http: / / dotlrn. org

7. ATutor: The Adaptive Technology Resource Centre (ATRC) at the University of Toronto Atutor, http: / / www . atutor.ca

8. Revuelta, P., Jiménez, J., Sánchez, J.M., Ruiz, B.: Multidevice system for Educational Accessibility of Hearing-Impaired Students. In: Proceedings of the $11^{\text {th }}$ IASTED Conference on Computer and Advanced Technology in Education (CATE), pp. 20-26 (2008)

9. Moreno, L., Iglesias, A., Castro, E., Martínez, P.: Using accessible digital resources for teaching database design: Towards an inclusive distance learning proposal. In: 13th Annual SIGCSE Conference on Innovation and Technology in Computer Science Education (ITiCSE 2008), pp. 32-36 (2008)

10. Brooke, J.: Sus: a 'quick and dirty' usability scale. In: Jordan, P.W., Thomas, B., Weerdmeester, B.A., McClelland, I.L. (eds.) Usability Evaluation in Industry, pp. 189-194. Taylor \& Francis, London (1996) 